\title{
Der Einfluss der Eingestuften Texte im Hinblick des Gemeinsamen Europäischen Referenzrahmens auf die Student*innenleistungen und die Nachhaltigkeit des Gelernten
}

\section{The Impact of the Leveled Texts Regarding the Common European Framework of Reference on the Performance and Retention of Undergraduate Students}

\author{
Ayşe ARSLAN ÇAVUŞOĞLU' (i), Fatih TEPEBAŞILI² [D
}

Arş. Gör. Dr., Necmettin Erbakan University, Ahmet Keleşoğlu Faculty of Education, Konya, Turkey

${ }^{2}$ Prof. Dr., Necmettin Erbakan University, Ahmet Keleşoğlu Faculty of Education, Konya, Turkey

ORCID: A.A.Ç. 0000-0001-9426-3722; F.T. 0000-0002-9412-2337

\section{Corresponding author:} Ayşe ARSLAN ÇAVUŞOĞLU, Necmettin Erbakan University, Ahmet Keleşoğlu Faculty of Education, Konya, Turkey

E-mail: aysearslancavusoglu@gmail.com

Submitted: 06.02 .2020

Revision Requested: 17.03.2020

Last Revision Received: 31.03 .2020

Accepted: 05.05.2020

Citation: Arslan Cavusoglu, A. \& Tepebasili, F. (2020). Der Einfluss der Eingestuften Texte im Hinblick des Gemeinsamen Europäischen Referenzrahmens auf die Student*innenleistungen und die Nachhaltigkeit des Gelernten. Alman Dili ve Edebiyatı Dergisi - Studien zur deutschen Sprache und Literatur, 43, 129-154. https://doi.org/10.26650/sdsl2020-0005

\begin{abstract}
DEUTSCH)
Bei der Einstufung und Bestimmung der Sprachniveaus hatten die Sprachkurse, die Schulbuchverlage und Sprachinstitute eigene Sprachkriterien, die von einem Land zu einem anderen oder von einem Sprachinstitut zu einem anderen variierten. Wegen dieser Variationen können die Stufen bzw. Sprachniveaus nicht übereinstimmen. Mit der Entstehung des Gemeinsamen Europäischen Referenzrahmens (GeR) konnten ein gemeinsamer Standard, gemeinsame Kriterien und eine gemeinsame Fremdsprachenunterrichtspolitik entwickelt werden. Man kann durch den Referenzrahmen die Sprachkenntnisse mit dem internationalen Standard festlegen. Und die bestätigten Sprachkenntnisse können in allen Ländern Europas anerkannt werden. Dadurch werden Sprachkenntnisse objektiv messbar, international vergleichbar sein. In dieser Studie wurde darauf abgezielt, den Beitrag derTexte, die im Hinblick des GeReingestuft sind, zu Leistungen der Sprachlernenden und zu Nachhaltigkeit des Gelernten zu bestimmen. Zu diesem Zweck nahmen 44 Student*innen (darunter 22 Männer und 22 Frauen), die an der Necmettin-ErbakanUniversität, Deutsch auf Lehramt studieren, an dieser Studie teil. 8 Wochen lang wurde die Experimentalgruppe mit den eingestuften Texten unterrichtet, während die Kontrollgruppe mit den Texten unterrichtet wurde, die nicht im Hinblick des GeR eingestuft sind. Ein Leistungstest wurde vor den Vorlesungen und nach den Vorlesungen durchgeführt. Die Textergebnisse bilden die Leistungsvortest- und Leistungsnachtestdaten. Um die Nachhaltigkeit des Gelernten zu messen, wurde nach 21 Tagen erneut ein Leistungstest durchgeführt. Das gemischte Modell vom ANOVA- Typ wurde für die Datenanalyse im Analyseprogramm R verwendet. Dem Forschungsergebnis zufolge zeigt die Experimentalgruppe einen signifikanten Unterschied im Zeitverlauf von der Kontrollgruppe.
\end{abstract}

Schlüsselwörter: eingestufte Texte, Der Gemeinsame Europäische Referenzrahmen, Leseverständnis, Leistungstest 


\begin{abstract}
ENGLISH)
When classifying and determining language levels, language courses, publishers and language institutes have their own language criteria, which vary from one country to another or from one language institute to another. Because of these variations, in the past, there were no standard language levels. With the emergence of the Common European Framework of Reference (CEFR), a common standard, common criteria and a common foreign language teaching policy could be developed. The framework can be used to determine language skills against the international standard. Moreover, the confirmed language skills can be recognized in all European countries. This will make language skills objectively measurable and internationally comparable. In this study, the influence of the leveled texts in respect of the Common European Framework of Reference on the achievement and retention of language learners in German reading lessons was measured quantitatively by comparing it with the non-leveled texts. Then it was supported with the students' views. Following this purpose, 44 students ( 22 males and 22 females), who are enrolled in the Department of Foreign Language Teaching of the University of Necmettin Erbakan, participated in this study. A post-test was applied to measure the change in students' performance after the 8-week course. To measure the permanence of the information, a retention test was performed again after 21 days. The data were analyzed through a mixed model ANOVA by using the R program. According to the results of the research, the experimental group showed a significant difference over time compared to the control group.

Keywords: Leveled texts, Common European Framework, reading comprehension, achievement test
\end{abstract}

\title{
EXTENDED ABSTRACT
}

Turkey participated in the Socrates project with the Council of Europe Decision (numbered 253/2000 / EC) on 24 January 2000 for a common policy in Europe. Foreign language teaching and second foreign language teaching policy in European countries have been accepted in Turkey too. Foreign language teaching in European countries is carried out inline with the educational policy of the "Department of Modern Languages of the Council of Europe". This unit, which seeks to develop a common standard, common criteria and a common foreign language teaching policy in Europe, prepares "The Common European Framework of Reference for Languages". Today, the foreign language teaching policy is implemented in all European countries based on this framework.This rating system is built on levels called A1, A2 (basic user), B1, B2 (independent user) and $\mathrm{C} 1, \mathrm{C} 2$ (proficient user). With this regulation, foreign language training materials are prepared accordingly.

Students should acquire four basic skills (reading, writing, listening and speaking) to master a foreign language. The acquisition of these four basic skills takes place through their integration and interdependence. The development of the act of speech first requires the development of listening comprehension. Also, improving listening skills depends on speaking ability. Likewise, the development of writing and reading skills also takes place in interaction with each other. On the whole, the combination of these four skills leads to mastering a foreign language. 
There are many studies aimed at teaching a foreign language effectively in terms of these four skills. But most of these studies are in English. Each language has its own reading strategies, as well as its own teaching and learning strategies. So far, only a limited number of research on reading in German exists in Turkey. Moreover, almost half of this research is theoretical.

Today, reading is an indispensable part of human life. By reading, one can think better, and, one can learn to form criticism and to accept criticism. Reading is a process in which signs and symbols are perceived by the eyes and ears, and then interpreted by the brain in an interpretive way. Through reading, people can access different sources of new information, events and experiences. Therefore, this competence includes learning, analysis and interpretation.

Because reading is so important when learning a foreign language, the students at the German Language Teaching Program receive two years of "reading skills" and "extended reading and writing skills" seminars. Therefore, reading is one of the most important parts of the curriculum. Students take part in reading lessons for a total of four semesters, but the reading comprehension level of the students is not satisfactory.

Therefore this study aimed to focus on improving students' reading skills. Considering that it can contribute to the development of reading skills, levelled texts prepared within the context of the Common European Framework for Languages were used as research material.

The impact of the leveled texts in respect of the Common European Framework of Reference on the achievement and retention of language learners in German reading lessons was measured quantitatively by comparing it with the non-leveled texts. Then it was supported with the students' views. Following this purpose, 44 students ( 22 males and 22 females), who are enrolled in the Department of Foreign Language Teaching of the University of Necmettin Erbakan, participated in this study. A success test was applied to measure the change in students' performance after the 8-week course. To measure the permanence of the information, a retention test was performed again after 21 days. Mixed model ANOVA was used for data analysis using the $\mathrm{R}$ program. The findings revealed that the experimental group showed a significant difference over time compared to the control group. 


\section{Einleitung}

Die Türkei hat an dem Sokrates-Projekt mit der Entscheidung des Europarates (nummeriert 253/2000 / EG) am 24. Januar 2000 für gemeinsame Fremdsprachenunterrichtspolitik in Europa teilgenommen'. Sprachplanung der europäischen Länder ist daher auch in der Türkei akzeptiert worden. Fremdsprachenunterricht in den europäischen Ländern wird entsprechend der Bildungspolitik von der „Abteilung für moderne Fremdsprachen des Europarates" vollzogen. Diese Abteilung, die einen gemeinsamen Standard, gemeinsame Kriterien und eine gemeinsame Fremdsprachenunterrichtspolitik in Europa entwickeln will, erstellt den „Gemeinsamen Europäischen Referenzrahmen für Sprachen". Heute wird die Fremdsprachenunterrichtspolitik in allen europäischen Ländern auf der Grundlage dieses Rahmenprogramms durchgeführt. Dieses Bewertungssystem baut sich auf Niveaus (A1, A2 (Anfänger), B1, B2 (Mittelstufe) und C1, C2 (Fortgeschrittene)) ${ }^{3}$ auf. Mit dieser Regelung werden fremdsprachige Schulungsmaterialien dementsprechend vorbereitet.

Weil Textlehre mit der Sprachlehre parallel ist, steht es immer in der Diskussion, was für einen Einfluss jede neue vorgebrachte Methode auf die Lesefertigkeiten ausüben kann und wie Texte effektiv im Sprachunterricht eingesetzt werden können.

Von derVergangenheit bis zur Gegenwart ist es zu bevorzugen, dass die im Sprachunterricht verwendeten Texte vom Leichten zum Schwierigeren, vom Einfachen zum Komplexen, vom Konkreten zum Abstrakten eingestuft worden sind (Dzaldov \& Petersen, 2005).

Bei der Einstufung und Bestimmung der Sprachniveaus hatten die Sprachkurse, die Schulbuchverlage und Sprachinstitutionen eigene Sprachkriterien. Die weit akzeptierte Hauptnivellierung war ähnlich wie heute Grund-, Mittel- und Oberstufe. Diese 3 Hauptstufen konnten in sich in mehrere Stufen eingeteilt werden. Auf jedem Niveau unterschieden sich die Sprachstrukturen, die Grammatik, der Wortschatz, die Themen und Inhalte voneinander, je nach den Sprachkursen, Buchverlagen oder Sprachinstitutionen, weil jede davon ihre eigenen Kriterien aufgestellt hat. So hatte jeder ein eigenes Einstufungssystem. Einige haben die Stufen mit Zahlen zum Ausdruck gebracht. Die Zahl 1 wurde als Schwelle akzeptiert. Entsprechend dem fortschreitenden Niveau hat sich die Zahl erhöht, die den Schwierigkeitsgrad vertritt. Andere haben die

1 http://adp.meb.gov.tr/nedir.php

2 Der Gemeinsame Europäische Referenzrahmen ist als Referenzrahmen oder GeR abgekürzt worden.

3 Europarat, Der Gemeinsame Europäische Referenzrahmen für Sprachen: Lernen, Lehren, Beurteilen, 2001, S. 35. 
Stufen mit Buchstaben beschrieben, die von A bis Z reichen, die den Schwierigkeitsgrad von leicht bis schwer einstufen. Weil diese Nivellierung von Einem zum Anderem variiert hat, konnte die Rede von Übereinstimmungen zwischen den Stufen nicht sein. Beispielsweise konnte ein Text auf der Mittelstufe in einem Sprachkurs, nahe dem Anfangsniveau in einer Sprachinstitution sein, weil kein gemeinsamer Referenzrahmen vorlag. Davon ging das Problem aus, inwieweit ein Sprachlerner die Sprache beherrschte und nach wem oder nach welchem Sprachkurs dieses Niveau festgelegt wurde.

Z.B sucht ein Arbeitgeber für seine Firma einen Arbeiter, der im Namen der Exportabteilung seines Unternehmens Telefongespräche führen soll. Vermutlich wird er den Bewerber auswählen, der die Sprache fließend beherrscht. Die Personen, die sich um die Stelle beworben haben, sagen aus, dass sie diese Sprache ganz gut beherschen würden.

Wie aber soll der Arbeitgeber beurteilen, wie gut diese Personen die sogenannte Fremdsprache beherrschen? Meinen diese Bewerber damit, dass sie fließend allägliche Umgangssprache sprechen, wie zum Beispiel beim Supermarkt etwas kaufen; einem Touristen den Weg zeigen und die Adresse sagen? Oder wünscht er als Arbeitgeber, dass sein Arbeiter so hervorragende Fremdsprachenkenntnisse hat, wie er Ertragskosten des Unternehmens gegenüber den Personen am Telefon mühelos und fließend sagen und danach darüber etwas aufschreiben und noch durch Telefongespräch an die Dritten weiterleiten soll? Also man braucht hier eine objektive Einstufung der Sprachkenntnisse. GeR bietet diese Einstufung nicht nur als einzelne Sprachen, sondern auch als einzelne Fertigkeiten wie Lesen, Schreiben, Hören und Sprechen. Man kann durch den Referenzrahmen die Sprachkenntnisse mit dem internationalen Standard festlegen. Und die bestätigten Sprachkenntnisse können in allen Ländern Europas anerkannt werden, und jeder, der sich damit auskennt, kann antworten; ;Ich spreche Deutsch auf dem Niveau B1 des GeR." oder "Ich schreibe französische Texte auf dem Niveau C2." Dadurch werden Sprachkenntnisse objektiv messbar, international vergleichbar sein. Der GeR bietet die Möglichkeit, Wettbewerber aus unterschiedlichen Ländern miteinander zu vergleichen. Deutsch auf dem Niveau B2 zu sprechen bedeutet nämlich für einen Spanier genau dasselbe wie für einen Schweden oder Türken ${ }^{4}$.

Ein anderes Problem bei der Einstufung der Texte nach verschiedenen Kriterien der unterschiedlichen Institutionen war, dass riesige Menge von Zeit, Geld und Energie dem Organisieren gewidmet wurde (Dzaldov \& Petersen, 2005, S. 221).

4 https://talkreal.org/blog/der-gemeinsame-europaeische-referenzrahmen-fuer-sprachen-ger/ Zugriffsdatum: 15.07.2018 
Trotzdem gab keines dieser Kriterien bei der Texteinstufung auf die Interessen, Motivationen, Hintergrunderfahrungen und -wissen oder die soziokulturellen Identitäten der Leser in Acht (Dzaldov \& Petersen, 2005, S. 223).

Trotz oben genannter Probleme sind die eingestuften Texte im Fremdsprachenunterricht in den Vordergrund gestellt worden und es ist nachgewiesen, dass die Texte, die laut dem Niveau der Studenten vorbereitet worden sind, die Studenten motiviert haben (Siehe: Dzaldov \& Petersen, 2005, S. 222; Glasswell \& Ford, 2010, S. 57).

Die oben erwähnten Fakten zeigen, dass ein großes Interesse an eingestuften Texten besteht. Darüber hinaus geht es um eine Einstufungsmanie (ein Begriff von Syzmusiak \& Sibborson, 2001; zitiert nach Dzaldov \& Petersen, 2005, S. 222). So haben Schulen, Institute bzw. Behörden für Bildungssysteme begonnen, nach ihren eigenen Kriterien die Texte einzustufen.

Mit der Ankündigung des GeR in 2001, im Europäischen Jahr der Sprachen, wurden große Schritte gemacht. Die Aneignung der gemeinsamen Kriterien bei der Fremdsprache bzw. beim Lesen hat ermöglicht, die Verschwendung von viel Zeit, Energie, Geld bei der Einstufung der Texte und die Unangemessenheit der Textinhalte, -struktur und -themen für die Fremdsprachenleser zu beheben.

Anstatt verschiedener Kriterien, die von einem Sprachkurs zu anderem variieren, ist ganz Europa mit dem Gemeinsamen Europäischen Referenzrahmen zu einem gemeinsamen Standard bzw. gemeinsamen Sprachniveaus übergegangen.

\section{Methode}

Dieser Teil belegt die geplante Methode der Forschung, die Stichprobe, Datenerhebungstechniken und -instrumente und die Analyse der Daten.

\subsection{Aufbau und Ziel der Forschung}

In dieser Studie wird der Beitrag der Texte zu Leistungen der Sprachlernenden und zu Nachhaltigkeit des Gelernten gemessen, die im Hinblick des GeR eingestuft sind. Darum werden die Teilnehmer*innen in zwei Gruppen eingeteilt (Experimentalgruppe und Kontrollgruppe), Leistungstests vollzogen, die Testergebnisse der Gruppen analysiert, 
miteinander verglichen und kommentiert. Mit den an der Experimentalgruppe teilnehmenden Student*innen werden Gespräche über die eingestuften Texte geführt. Auf diese Weise können die Vor- und Nachteile der eingestuften Texte im Hinblick des GeR ans Licht gebracht werden.

\subsection{Forschungsmodelle}

Die vorliegende Studie basiert auf der quantitativen Forschung. Sie wurde auf einer Grundlage von einem randomisierten Experimentdesign aufgebaut, in dem sich Vor- und Nachtest, Kontroll- und Experimentalgruppe befinden, um die Leistungen der Student*innen zum Lesen in deutscher Sprache und die Nachhaltigkeit des Gelernten zu bestimmen.

Durch dieses Design sind die Veränderungen im Verhalten der Versuchsperson (abhängige Variable) eindeutig auf die bewusste Veränderung der unabhängigen Variablen (Treatment) zurückzuführen.

Nach Büyüköztürk und seinen Kollegen (2012, S. 205) steht dieses Design in einem Zusammenhang, weil dieselben Personen zweimal über die abhängige Variable gemessen werden. Es steht aber auch in keinem Zusammenhang, weil die Messungen der aus verschiedenen Probanden gebildeten Experimental- und Kontrollgruppen verglichen werden. In dieser Forschung werden die Teilnehmer*innen in der Experimentalgruppe und Kontrollgruppe dreimal (Vortest-Nachtest-verzögerter Nachtest) über die abhängige Variable (Leistungen) gemessen. Es steht aber auch in keinem Zusammenhang, weil die Teilnehmer*innen in den Gruppen aus verschiedenen Probanden bestehen.

Die symbolische Ansicht des experimentellen Modells ist wie folgt (Karasar, 2009, S. 97):

Tabelle 2. das experimentelle Model der Studie

\begin{tabular}{|l|l|l|l|l|l|l|}
\hline G1(Experiment) & R & Q1.1 & X & Q1.2 & Q1.3 & Schriftliche Stellungnahme \\
\hline G2 (Kontrolle) & R & Q2.1 & & Q2.2 & Q2.3 & \\
\hline G1: Die Experimentgruppe, die eingestufte Texte bearbeiten wird. \\
G2: Die Kontrollgruppe, die zufällig ausgewählte Texte bearbeiten wird. \\
R: Die zufällige Zuweisung der Probanden \\
X: Unterrichtsbearbeitung mit eingestuften Texten im Hinblick des GeR \\
Q1.1- Q2.1: Vortest \\
Q1.2- Q2.2: Nachtest \\
Q1.3- Q2.3: Verzögerter Nachtest \\
\hline
\end{tabular}




\subsection{Teilnehmer*innen}

Der Versuch wurde im akademischen Jahr 2017-2018 durchgeführt An der Forschung haben 44 Deutschlehrerkandidat*innen teilgenommen, die an der Necmettin Erbakan Universität, Deutsch auf Lehramt studieren. Sie waren im ersten Semester des vier jährigen Studiums. Der Grund, warum die Student*innen im ersten Semester ausgewählt wurden, ist, dass sie im ersten Studienjahr das Seminar für Lesefertigkeit und im zweiten Studienjahr das Seminar für erweiterte Lesefertigkeit erhalten. Das Curriculum enthält kein Leseseminar im dritten und vierten Studienjahr.

\subsubsection{Bestimmung des Leseverständnisniveaus der Teilnehmer*innen}

Vor der Ausführung des LV-Vortests (Leistungstest) wurden die Probanden einem von dem Goethe Institut versorgten Einstufungstest ${ }^{5}$ auf dem Niveau A2-B1 unterzogen, um ihre Niveaus zum Leseverstehen zu messen. Für den Einstufungstest zum Lesen von Deutschen Texten hatten die Student*innen 65 Minuten.

Weil das Goethe Institut eine weltweit bekannte Kooperation für Deutschlehren und -lernen ist und viele Zuständige für Prüfungs-, Beurteilungs- und Evaluierungsprozess hat, sind die wissenschaftlichen Kriterien der Gültigkeit und Zuverlässigkeit der Prüfungsfragen nicht erneut überprüft worden.

Tabelle 3. Einstufungstestscoren der Teilnehmer*innen

\begin{tabular}{|l|l|l|l|l|l|l|l|l|l|l|}
\hline T & N & M & Sd & Median & $\begin{array}{l}\text { Getrimter } \\
\text { Mittelwert }\end{array}$ & Mmin & Mmax & Range & Ske. & Kurt. \\
\hline B1 & 44 & 52.27 & 12.78 & 55 & 51.81 & 30 & 80 & 50 & 0.25 & -1.00 \\
\hline A2 & 44 & 87.23 & 9.22 & 88 & 88.06 & 68 & 100 & 32 & -0.78 & -0.46 \\
\hline $\begin{array}{l}\text { N: Teilnehmeranzahl. Sd: Standartabweichung } \\
\text { T: Testniveau M: Mittelwert }\end{array}$
\end{tabular}

Die Testscoren zeigen, dass es sich um eine normale Verteilung sowohl beim Einstufungstest A2 als auch B1 handelt. Die Mittelwerte (52.27 für B1 und 87.23 für A2) und die Medianwerte (55 für B1 und 88 für A2) stehen näher.

5 Die Antworten auf die Gültigkeit und Zuverlässigkeit der Prüfungsfragen können auf der Webseite des Goetheinstituts https://www.goethe.de/de/spr/kup/prf/qua.html https://www.goethe.de/de/spr/ kup/prf/ koo.html gefunden werden. Zugriffsdatum: 20.05.2018 
Nebenher liegen die getrimmten Mittelwerte (51.81 für B1 und 88.06 für A2) nahe an den Mittelwerten. Burkhart \& Sedlmeier drücken aus, dass der getrimmte Mittelwert einen bestimmten Anteil der Daten berücksichtigt, wenn der arithmetische Mittelwert durch Extremwerte (Ausreißer) stark beeinflusst wird (2015, S. 34). Nach dem oben angegebenen getrimmten Mittelwert ist es klar zu sagen, dass es bei der Verteilung der Einstufungstestscoren um fast keinen Ausreißer geht. So verteilen sich die Student*innenleseverständnisniveaus nicht weit voneinander.

Ein anderer Hinweis der normalen Verteilung sind die Werte der Skewness und Kurtosis. (Ske: 0.25 und Kurt: - 1.00 für A2 Test und ske: 0.78 und kurt: -0.46 für B1 Test). Die Werte für Schiefe (Skewness) und Kurtosis zwischen -2 und +2 gelten als akzeptabel, um die normale univariate Verteilung zu beweisen (George \& Mallery, 2010). So ist die Schiefe für eine Normalverteilung Null, und alle symmetrischen Daten sollten eine Schiefe nahe Null aufweisen.

So lässt es sich aus den obigen Tabellen erkennen, dass die Erstklässler*innen des vier jährigen Studiums erfolgreich auf dem Leseverständnisniveau A2 sind. Aber sie scheitern auf dem Niveau B1.

Nach dem Einstufungstest und der Analyse der Einstufungstests wurden die Teilnehmer*innen in zwei Gruppen als Experimentalgruppe und und Kontrollgruppe eingeteilt.

\subsubsection{Die Verteilung der Probanden}

Die meisten Student*innen haben ein Jahr lang vor dem Fachstudium in der Vorbereitungsklasse studiert. Sie wurden mit dem Lehrbuch „Menschen“ unterrichtet.

Fast ein Viertel der Probanden sind die Student*innen, die bereits eine Erfahrung in einem deutschsprachigen Raum hatten, eine Befähigungsprüfung (Befreiungsprüfung für die Vorbereitungsklasse) am Anfang des Studienjahres abgelegt und mit ausreichenden Sprachkenntnissen ihr Studium an der Fakultät begonnen haben, ohne am Vorbereitungsprogramm teilnehmen zu müssen. Abgesehen von diesem Unterschied wurden die Student*innen zufällig aufgeteilt, weil sowohl die in der Vorbereitungsklasse ein Jahr lang studierten Student*innen, als auch die Student*innen, die Befreiungsprüfung für die Vorbereitungsklasse bestanden haben (keine Vorbereitungsklasse besucht haben), auf dem gleichen Niveau gezählt wurden und sie ähnliche Testscoren erhalten haben. 
Tabelle 4. Verteilung der Probanden

\begin{tabular}{|l|l|l|l|l|l|}
\hline Gruppe & $\begin{array}{l}\text { weibliche } \\
\text { Probanden }\end{array}$ & $\%$ & $\begin{array}{l}\text { männliche } \\
\text { Probanden }\end{array}$ & $\%$ & $\begin{array}{l}\text { Gesamte } \\
\text { Teilnehmer }\end{array}$ \\
\hline Kontrolle & 19 & 86 & 3 & 14 & 22 \\
\hline Experimentell & 20 & 90 & 2 & 10 & 22 \\
\hline
\end{tabular}

\subsubsection{Leistungsvortestscoren der Teilnehmer*innen}

Die Auswertung der Teilnehmer*innen in Bezug auf Leistungs-Vortestscoren ist in der folgenden Tabelle angegeben. Nach der Datenerhebung wurden Student*innenmeinungen von der Experimentalgruppe zu den eingestuften Texten erhalten.

Tabelle 5. Leseverständnis--Vortestscoren der Teilnehmer*innen

\begin{tabular}{|l|l|l|l|l|l|l|l|}
\hline & $\mathrm{N}$ & $\mathrm{X}$ & Konfidenzintervall von 95 Prozent & Der Mittelwert von Differenzen & $\mathrm{F}$ & $\mathrm{T}$ & $\mathrm{P}$ \\
\hline $\mathrm{G} 1$ & 22 & 51.8 & \multirow{2}{*}{$-5.49294912 .765676$} & 3.7 & 42 & 0.80384 & 0.426 \\
\hline $\mathrm{G} 2$ & 22 & 48.1 & & & \\
\hline
\end{tabular}

Die Daten durch Analyse von t-Test für LV-Vortestscoren der Teilnehmer*innen zeigen, dass der Mittelwert der Leseverständnisscoren der Experimentalgruppe als 51.8 und der Kontrollgruppe als 48.1 zum Vorschein gekommen ist. Aus dem Ergebnis der Testanalyse ist der t-Wert als -0.80384, und der p-Wert als 0.426. Es kann entsprechend den Analyseergebnissen $t=-0.80384, p=0 . .426 p>0,05$ erklärt werden, dass es kein signifikanter Unterschied zwischen den beiden Gruppen entsteht und die Gruppen auf dem gleichen Leseverständnisniveau sind.

\subsection{Datenerhebung}

Für die Bestimmung ihrer Leistungen zum Lesen von Deutschen Texten wurde jede Gruppe vor und nach dem Versuch aufgefordert, die von Goethe Institut versorgten Prüfungen abzulegen, um herauszufinden, ob die eingestuften Texte auf Leistungen und die Nachhaltigkeit des Gelernten von den Student*innen Einfluss ausüben könnten. Die Studierenden sollten 20 Multiple-Choice-Fragen, die aus 3 verschiedenen Aufgabentypen bestehen, zum Leseverständnis beantworten. Letztlich wurden den Probanden der 
Experimentalgruppe schriftliche Fragen gestellt, um ihre Meinungen über die eingestuften Texte schriftlich zu erfassen.

Tabelle 6. Die Datenerhebungsmethoden und Materialien der Experimentalgruppe und Kontrollgruppe

\begin{tabular}{|l|l|l|l|l|}
\hline \multicolumn{5}{|c|}{ Die Datenerhebungsprozesse der Experimentalgruppe } \\
\hline & $\begin{array}{l}\text { Vor } \\
\text { dem Versuch }\end{array}$ & $\begin{array}{l}\text { Während } \\
\text { des Versuchs }\end{array}$ & $\begin{array}{l}\text { Nach } \\
\text { dem Versuch }\end{array}$ & $\begin{array}{l}\text { 3 Wochen } \\
\text { dem Versuch }\end{array}$ \\
\hline Einstufungstest & & & & \\
\hline Leistungstest & $*$ & & $*$ & $*$ \\
\hline Schriftliche Stellungnahme & $*$ & & $*$ & \\
\hline $\begin{array}{l}\text { 8 Wochen lang behandelte } \\
\text { eingestufte Texte } \\
\text { auf dem Niveau B1 }\end{array}$ & & $*$ & & \\
\hline \multicolumn{2}{|l|}{ Die Datenerhebungsprozesse der Kontrollgruppe } & \\
\hline Einstufungstest & & & & \\
\hline Leistungstest & $*$ & & $*$ & $*$ \\
\hline $\begin{array}{l}\text { 8 Wochen lang behandelte } \\
\text { Texte }\end{array}$ & $*$ & & & \\
\hline
\end{tabular}

\subsubsection{Einstufungstest}

Bei dieser Forschungsphase wurde erforscht, einen angemessenen Einstufungstest für die Bestimmung und Bewertung der Student*innenniveaus zu finden. Weil sich das Hauptthema der vorliegenden Arbeit auf den Gemeinsamen Europäischen Referenzrahmen für Sprachen bezieht, wurde bei der Auswahl der Leseverständnisteste Rücksicht auf den GeR genommen. Intensive Gespräche mit den Lehrkräften in der Sprachhochschule an der Necmettin Erbakan Universität wurden geführt, um festzustellen, welches Niveau, die von dem GeR vorgelegten 6 Hauptniveaus A1, A2, B1, B2, C1 und C2 in Acht genommen wurde, um die Niveaus der Erstklässler*innen zu bestimmen. Laut den Aussagen der Lehrkräfte hätten die Student*innen am Ende der Vorbereitungsklasse das B1- Niveau erreicht. Die Aussagen der Lehrenden über die Vorbereitungsklasse und die 3 Monate Sommerferien vor Auge haltend, wurde beschlossen, dass ein Einstufungstest erst auf dem Niveau A2, und dann nach dem Ergebnis dieser Prüfung, noch ein B1 Test durchgeführt wird. Für die Einstufungsteste auf dem Niveau A2 und B1 wurde mit dem Goethe Institut-Ankara Kontakt aufgenommen. Nach dem Erhalt der Teste wurde zuerst der Einstufungstest für Leseverstehen auf dem Niveau A2 durchgeführt. Wie es in der Tabelle erkennbar ist, schlossen die Student*innen den Test erfolgreich ab. Dann wurde

6 Einstufungstest wurde vor der Aufteilung der Klasse in zwei Gruppen durchgeführt. 
der Test B1 abgelegt. Es ist in der Tabelle offengelegt, dass die Student*innen auf dem Niveau B1 scheitern. Dementsprechend kann ausgesagt werden, dass die Erstklässler*innen auf dem A2 Leseverständnisniveau waren.

\subsubsection{Leistungstest (Leseverständnistest)}

Im weiteren Verlauf ließ es sich entscheiden, dass sich die Probanden in der Experimentalgruppe mit den B1 Texten im Hinblick des GeR und die Probanden in der Kontrollgruppe mit den Texten auseinandersetzen, die nicht nach dem GeR eingestuft sind.

Nach der Bestimmung der Student*innenniveaus wurden die Teilnehmer*innen in Kontrollgruppe und Experimentalgruppe aufgeteilt.

Nach der Aufteilung der Teilnehmer*innen haben die Student*innen einen von dem Goethe Institut versorgten LV-Leistungstest auf dem Niveau B1 abgelegt. Diese Ergebnisse haben die Leistungsvortestdaten gebildet. Fast ein Semester lang (8Texte für 8 Woche) hat die Experimentalgruppe die eingestuften Texte im Hinblick des GeR bearbeitet, während die Kontrollgruppe die zufällig ausgewählten Texte aus den Büchern für die Mittelstufe bearbeitet hat, die nicht im Hinblick des GeR eingestuft sind. Am Ende der 8 Wochen (nach den Seminaren) wurde der gleiche Leistungstest bei den beiden Gruppen durchgeführt, dessen Ergebnisse die Nachtestdaten bilden. Nach dem Nachtest machten die Student*innen Semesterferien. 21 Tage lang besuchten sie keine Vorlesung. Nach 3 Wochen, am Anfang des zweiten Semesters, wurden die Student*innen dem gleichen Leistungstest unterzogen, dessen Ergebnisse die Daten des verzögerten Nachtests bilden.

\subsubsection{Schriftliche Stellungannahme}

Am Ende des Semesters, bzw. am Schluss des Versuchs wurden die Student*innen in der Experimentalgruppe nach ihren Meinungen über die behandelten eingestuften Texte gefragt. Bei der Stellungnahme der Student*innen in der Experimentalgruppe wurde danach gefragt, ob die Strukturen, der Wortschatz und die Inhalte der eingestuften Texte anders sind als die Texte, die sie bisher gelesen haben. Die Rückmeldungen der Probanden wurden zur Unterstützung der quantitativen Daten hinzugefügt und als Teil des experimentalen Modells wurde der empirische Teil der vorliegenden Forschung vervollständigt. 


\subsection{Datenanalyse der Forschung}

Die quantitativen Daten der Forschung wurden in Analyseprogramm $R$ und SAS (Universität-Ausgabe) mit den geeigneten Tests (ein gemischtes Modell vom ANOVA-Typ und t-Tests) analysiert. Weil t-Test zusätzlich Gleichheit der Varianzen voraussetzte, wurde es mittels Levene-Tests untersucht. Nach dem Levene-test wurde der t-Test sowohl am Anfang als auch am Ende der Forschung durchgeführt, da die Stichproben unverbunden und normalverteilt sind. Zum Schluss der Forschung wurden die Daten mit dem gemischten Modell vom ANOVA- Typ analysiert, um zu bestimmen, ob über einen Zeitraum von 8 Wochen ein bedeutsamer Unterschied zwischen der Experimental- und Kontrollgruppe entsteht. Übrigens wurden Boxplots und Grafiken erstellt, um die Forschungsresultate deutlicher zu machen.

Die schriftlichen Antworten der Probanden in der Experimentalgruppe auf die Fragen „Gibt es einen Unterschied zwischen den eingestuften Texten und den bisher gelesenen Texten? Wenn ja, was sind die Unterschiede?" wurden durch die deskriptive Analyse ausgewertet. Die deskriptive Analyse ist die einfachste Form der Analyse. Es ist eine Untersuchung der Daten, wie sie gezeigt, dargestellt, beschrieben und übertragen werden. Es geht also um keine detaillierte und auf Theorie zurückführende Zerlegung (Sönmez \& Alacapınar, 2014, S. 244). Gemäß diesem Ansatz werden die Daten in Übereinstimmung mit vorher bestimmten Kategorien interpretiert.

\subsection{Durchführungsphase der Forschung}

In dieser Studie wurden die Lesetexte mit den Student*innen im Wintersemester des vier jährigen Studiums im Rahmen des Seminars Lesefertigkeit I bearbeitet. Während die Experimentalgruppe die eingestuften Texte behandelt hat, hat die Kontrollgruppe die Texte bearbeitet, deren Niveaus nicht nach den Kriterien des GeR bestimmt sind. Die Seminare haben ein Semester lang gedauert. Weil die Vor- und Nachteste innerhalb der Vorlesung durchgeführt wurden, konnten die Texte nur noch 8 Wochen lang behandelt werden.

Für beide Gruppen wurde als Unterrichtansatz ein „Handlungsorientierter Ansatz" hinsichtlich des GeR bestimmt. Das gleiche didaktisch- methodische Konzept wurde für die beiden Gruppen durchgeführt. Auf diese Weise differenziert nur das Unterrichtsmaterial, um den Unterschied der Wirkung der eingestuften Texte von anderen Texten hervorzuheben. 
Beim handlungsorientierten Unterricht steht die Interaktion zwischen dem Lehrer und den Student*innen im Vordergrund. Der Lehrer ist als Lernpartner tätig. Er steuert die Student*innen und fördert sie zum selbstbestimmten Lernen. Als Ergebnis dieser Zusammenwirkung tritt schriftlich oder mündlich ein sprachliches Produkt auf'. Bei der handlungsorientierten Methode sollen mit der Aussage von Pestalozzi „Kopf, Herz und Hand" also kognitives, affektives und psychomotorisches Lernen der Schüler in ein richtiges Verhältnis, die Anteilnahme beider Seiten berücksichtigend zueinander gebracht werden (Kuwan \& Waschbüsch, 1999, S. 58; Meyer, 1987, S. 393).

Auch bei der Themenwahl der Texte der Kontroll- und Experimentalgruppe wurde auf das Alter, Geschlecht und die Interessen der Student*innen geachtet.

Hier werden die wöchentlichen Themen aufgezeigt;

Tabelle 7. wöchentliche Themen

\begin{tabular}{|c|c|c|}
\hline \multicolumn{3}{|c|}{ Experimentalgruppe } \\
\hline Woche & Datum & Thema \\
\hline 1. Woche & 13.10 .2017 & Keine Lust Auf Schule \\
\hline 2. Woche & 20.10 .2017 & Macht Kaugummi Schlau? \\
\hline 3. Woche & 27.10 .2017 & Kinderarmut- Einmal Arm Immer Arm \\
\hline 4. Woche & 03.11 .2017 & Geteiltes Auto/ Geteilte Kosten \\
\hline 5. Woche & 10.11.2017 & Ist Daddeln Gefährlich? \\
\hline 6. Woche & 17.11.2017 & Prothesen Dürfen bunt sein: Schaut ruhig hin \\
\hline 7. Woche & 01.12 .2017 & Erfolgreiche Anti-Rauch-Aktion \\
\hline 8. Woche & 08.12 .2017 & Auch Clown Sein Wird Gelernt Sein \\
\hline \multicolumn{3}{|c|}{ Kontrollgruppe } \\
\hline 1. Woche & 10.10.2017 & Glück Im Cafe \\
\hline 2. Woche & 17.10 .2017 & Shakiras großes Herz \\
\hline 3. Woche & 24.10.2017 & Gleiches Geld für Frauen \\
\hline 4. Woche & 31.11 .2017 & Jürgen macht neue Bekanntschaften \\
\hline 5. Woche & 7.11 .2017 & Ein Kommunalpolitiker zum Thema "Single" \\
\hline 6. Woche & 14.11 .2017 & Wenn Computer Spiele zur Sucht wird. \\
\hline 7. Woche & 28.11 .2017 & Mein Vater \\
\hline 8. Woche & 05.12 .2017 & Danni \\
\hline
\end{tabular}

In der folgenden Tabelle ist ein Beispiel zu zeigen, wie jeder Unterricht aufgebaut ist.

7 Der Gemeinsame Europäische Referenzrahmen ist als Referenzrahmen, 2001. 


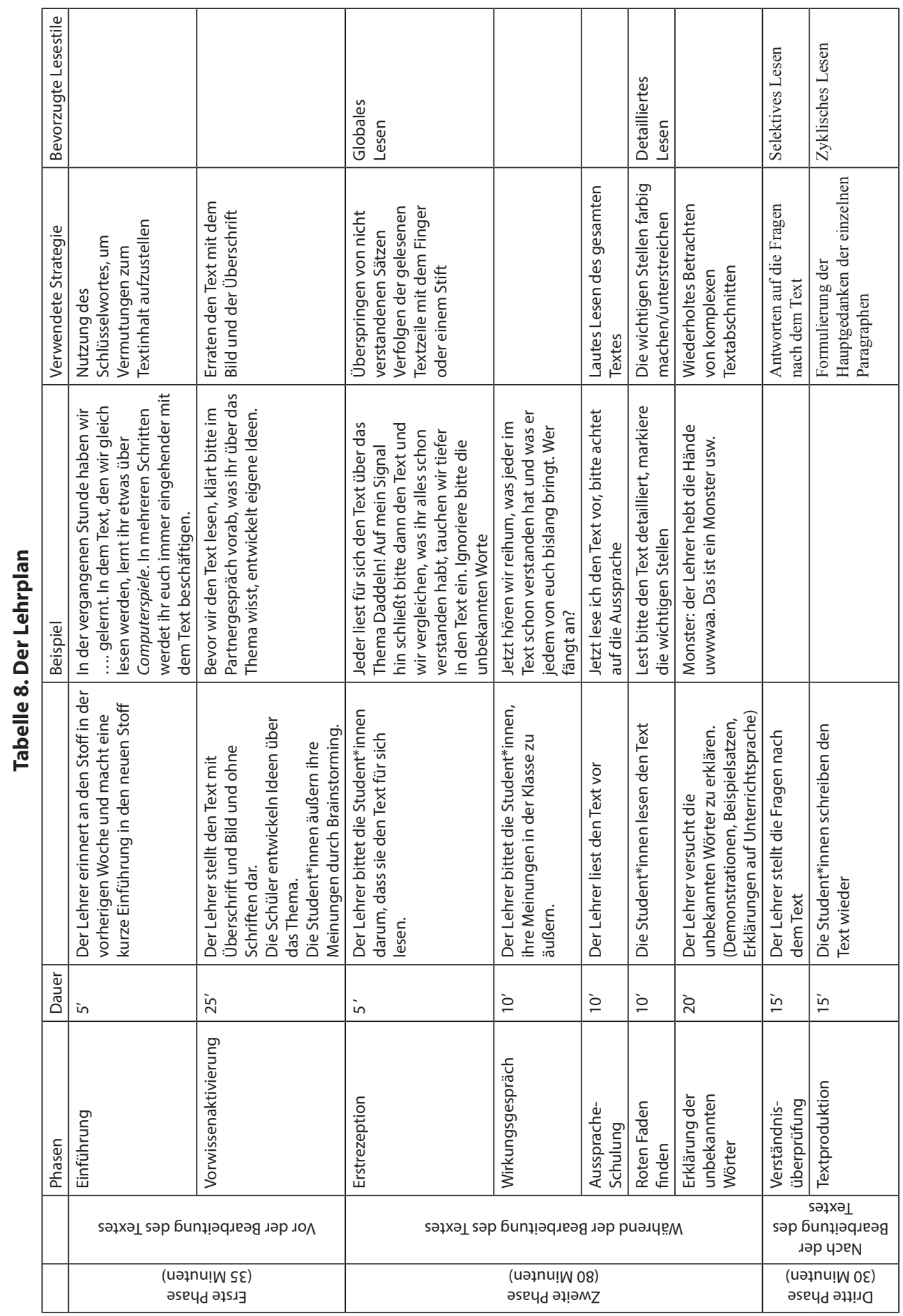


Wenn der oben angeführte Lehrplan zu untersuchen ist, ist es ersichtlich, zu sagen, dass in den beiden Gruppen die notwendigen Lesestrategien, Lesestilen und Leseaktivitäten so viel wie möglich angestrebt werden, um einen effektiven Leseunterricht durchzuführen.

\section{Befunde Und Kommentare}

In diesem Teil werden die Ergebnisse der statistischen Analyse der erhaltenen Daten zur Lösung der Forschungsprobleme dargestellt.

\subsection{ANOVA-Ergebnisse der Leistungstestscoren}

Die Student*innen in den beiden Gruppen wurden vor dem Versuch, nach dem Versuch und 21 Tage nach dem Versuch dem gleichen Test unterzogen. So hat sich die statistische Tendenz im Laufe der Zeit zum Vorschein bringen lassen.

Weil es sich bei den Daten der vorliegenden Untersuchung um einen Innersubjektfaktor (Zeit) und einen Zwischensubjektfaktor (Gruppen) handelte, wurde ein gemischtes Modell vom ANOVA-Typ genutzt. Im Übrigen wurde LSM (Least Squares Means) zur Analyse der Daten genutzt, um herauszufinden, aus welcher Gruppe der Unterschied stammt, wenn die Rede von einem signifikanten Unterschied ist.

Tabelle 9. ANOVA-Ergebnisse der Leistungstestscoren

\begin{tabular}{|l|l|l|l|l|l|}
\hline Effekt & SSn & SSd & F & $\mathrm{p}<.05$ & ges \\
\hline Gruppe & 1037.1212 & 19714.394 & 2.209507 & $1.446314 \mathrm{e}-01$ & 0.040621940 \\
\hline Zeit & 5728.4091 & 4779.545 & 50.338088 & $1.063692 \mathrm{e}-08^{*}$ & 0.189542156 \\
\hline Gruppe: Zeit & 192.0455 & 4779.545 & 1.687589 & $2.010056 \mathrm{e}-01$ & 0.007779534 \\
\hline
\end{tabular}

Tabelle 10. (Least Squares Means) LSM-Ergebnisse

\begin{tabular}{|l|l|l|l|l|l|l|l|}
\hline Simple Effect Level & Group & Group & Estimate & Standard Error & DF & t Value & $\mathrm{p}<.05$ \\
\hline Vortest & Experiment & Kontrolle & 3.6364 & 4.4597 & 84 & 0.82 & 0.4172 \\
\hline Nachtest & Experiment & Kontrolle & 3.6364 & 4.4597 & 84 & 0.82 & 0.4172 \\
\hline $\begin{array}{l}\text { Verzögerter } \\
\text { Nachtest }\end{array}$ & Experiment & Kontrolle & 9.5455 & 4.4597 & 84 & 2.14 & $0.0352^{*}$ \\
\hline
\end{tabular}

Nach den obigen ANOVA- und LSM-Ergebnissen weisen die oben angegebenen Werte darauf hin, dass die beiden Gruppen im Vor- und Nachtest gleichzeitig Fortschritte 
verzeichnet haben. Aber im verzögerten Nachtest ist ein bedeutsamer Unterschied zugunsten der Experimentalgruppe entsanden $\left(p<.05 .=0.0352^{*}\right)$.

Tabelle 11. Analysevariablen

\begin{tabular}{|l|l|l|l|l|l|l|l|l|}
\hline \multicolumn{10}{|c|}{ Analysevariablen } \\
\hline Zeit/Tests & Gruppe & N Obs & N & Mittelwert & Std Dev & Median & $\begin{array}{l}\text { Unteres } \\
\text { Quartil }\end{array}$ & $\begin{array}{l}\text { Oberes } \\
\text { Quartil }\end{array}$ \\
\hline 1/Nortest & Experiment & 22 & 22 & 51.82 & 12.96 & 52.50 & 40.00 & 60.0 \\
& Kontrolle & 22 & 22 & 48.18 & 16.80 & 50.00 & 35.00 & 55.0 \\
\hline 2/Nachtest & Experiment & 22 & 22 & 58.86 & 16.47 & 55.00 & 50.00 & 70.0 \\
& Kontrolle & 22 & 22 & 55.23 & 14.27 & 55.00 & 45.00 & 60.0 \\
\hline 3/Verzögerter & Experiment & 22 & 22 & 70.91 & 3.42 & 70.00 & 65.00 & 80.0 \\
Nachtest & Kontrolle & 22 & 22 & 61.36 & 14.41 & 65.00 & 45.00 & 70.0 \\
\hline
\end{tabular}

Wenn die Ergebnisse der Vor-, Nach- und verzögerten Teste miteinander verglichen werden, ist die Zunahme der Leistungspunkte in beiden Gruppen augenfällig. So haben die beiden Gruppen gewisse Fortschritte gemacht. Wenn die Werte im Vortest und Nachtest der Gruppen miteinander verglichen werden, kann gesagt werden, dass es um eine Fortentwicklung in jeder Gruppe geht. Während es um keine Überlegenheit im Vortest und Nachttest geht, handelt es sich um eine Überlegenheit im verzögerten Nachtest zugunsten der Experimentalgruppe.

In Folgenden werden die Grafiken zur Veranschaulichung der Daten hilfreich sein.
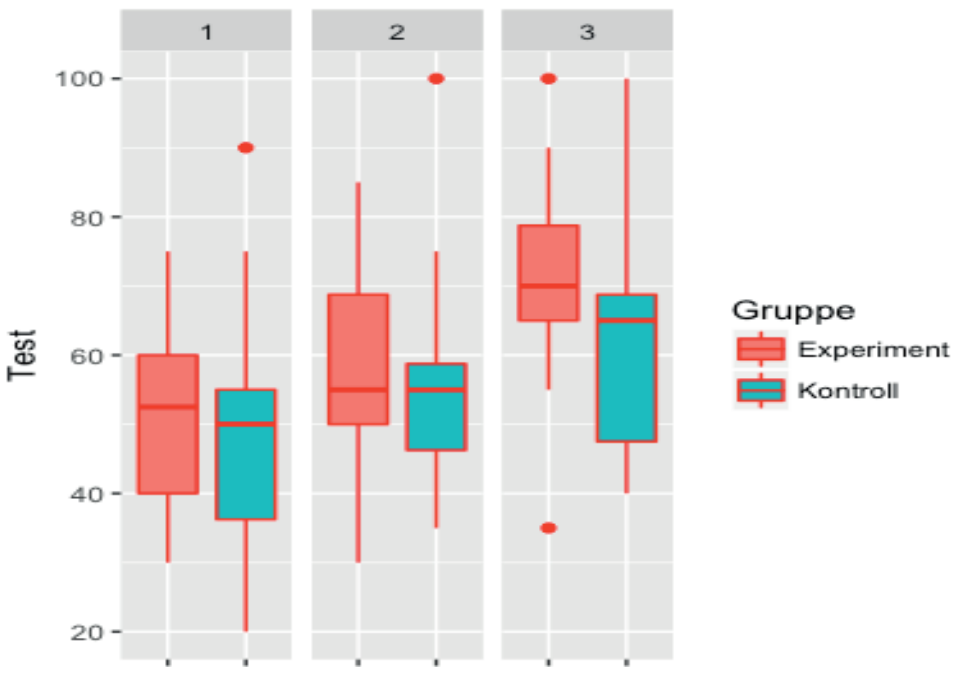

Abbildung 1. Boxplot der Leistungstests der Experimental- und Kontrollgruppe 
Im obigen Boxplot fallen drei verschiedene Testergebnisse auf, das untere Quartil, das als der kleinste Wert der Datenreihe definiert ist, ist im Vortest der Kontrollgruppe 35, im Vortest der Experimentalgruppe 40. Das oberste Quartil ist im Vortest der Kontrollgruppe 55, im Vortest der Experimentalgruppe 60.

Jede Gruppe hat nach 8 wöchigem Unterricht ihre Leistungsscoren um ca. 10 Punkte erhöht. So liegt im Nachtest der Kontrollgruppe der untere Quartilwert bei 45 und der obere Quartilwert bei 60 Punkten. Während die unteren und oberen arithmetischen Werte im Nachtest der Experimentalgruppe 50 und 70 sind.

Der dritte Test, in dem die Experimentalgruppe eine Überlegenheit gezeigt hat, sind die kleinsten und grössten arithmetischen Werte der Kontrollgruppe 45 und 70 und der Experimentalgruppe 65 und 80.

Nach diesen Scoren ist die Zunahme der Leistungspunkte besonders im verzögerten Test zugunsten der Experimentalgruppe deutlich erkennbar.

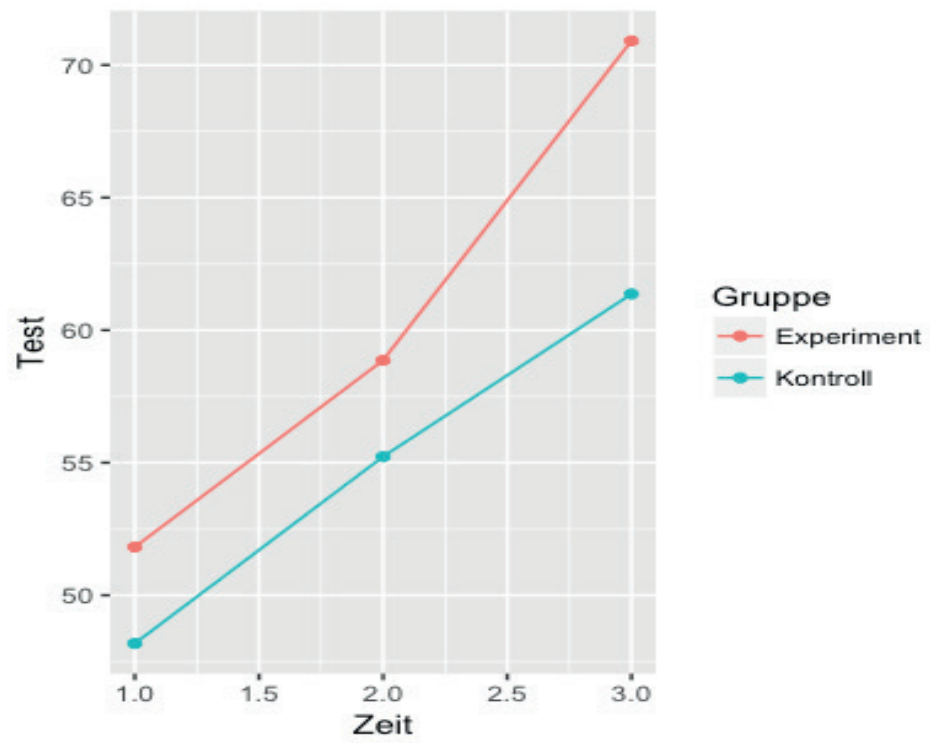

Abbildung 2. Interaktionsplot der Leistungstests der Experimental- und Kontrollgruppe

Das obige Interaktionsplot zeigt, dass der Mittelwert der Leistungspunkte von Experimentalgruppe im Vortest 52 und im Nachtest ungefähr 59, im verzögerten Test knapp unter 75 ist. Die Kontrollgruppe, erreicht zu Beginn etwa 48, im Nachtest knapp 
über 55 und im letzten Test, im verzögerten Test, circa 62. Wenn die beiden Gruppen miteinander verglichen werden, ergab sich ein deutlicher Unterschied zugunsten der Experimentalgruppe.

\subsection{Analysenergebnisse der Stellungnahmen der Experimentalgruppe ${ }^{8}$}

In diesem Teil sind die Antworten auf die Frage, ob es einen Unterschied zwischen den eingestuften Texten und bisher gelesenen Texten gibt, untersucht worden. In diesem Rahmen wurde von den Student*innen verlangt, ihre Meinungen schriftlich zu äußern.

Sowie Yıldırım und Şimşek (2008) in ihren Werken ausgedrückt haben, kann die Analyse der schriftlichen Dokumente alleine eine Datenerhebungsmethode sein, oder sie kann auch zur Unterstützung für die aus der Beobachtung oder dem Interview erhaltenen Informationen verwendet werden. In diesem Zusammenhang leisteten die Student*innenaufsätze einen großen Beitrag zur Gestaltung dieses Teiles.

Gemäß den Student*innenmeinungen kann gesagt werden, dass sie im Allgemeinen positive Meinungen zu den eingestuften Texten im Vergleich zu den anderen Texten haben. Nebenbei beeinflussen die für das Niveau der Student*innen angemessenen Texte durch ein höheres Maß die Student*innenleistungen. Außerdem haben die meisten Student*innen in Worte gefasst, dass sie beim Lesen vielen neuen bzw. unbekannten Wörtern begegnen und deswegen Schwierigkeiten beim Leseverstehen haben.

Die Antworten der Student*innen auf die Frage „Wenn ja, was sind die Unterschiede?" können nach verschiedenen Themen wie Aktualität, Wortstruktur und Inhalt gruppiert werden.

Hier sind die Student*innenmeinungen in einer Tabelle angegeben.

Tabelle 12. Der Prozent und die Frequenz der Student*innenmeinungen zu „eingestufte Texte“.

\begin{tabular}{|l|c|c|}
\hline & $f$ & $\%$ \\
\hline positiver Unterschied & 14 & 63.64 \\
\hline negativer Unterschied & 4 & 18.18 \\
\hline kein Unterschied & 4 & 18.18 \\
\hline
\end{tabular}

8 Weil in diesem Teil der Studie von einer umfangreichen qualitativen Forschung nicht die Rede ist, wurde die Analyse der Student*innenmeinungen mit dem quantitativen Teil bearbeitet und kurz vorgestellt, um die quantitativen Daten zu unterstützen. 
Die Student*innen, die positive Meinungen zu den eingestuften Texten gebildet haben, haben erläutert, dass die Texte sehr aktuell und authentisch und ihr Inhalt und ihre Satzstruktur verständlicher als andere Texte sind. Einige Beispiele;

Die Studentin F. Ş.: „Der Unterschied der eingestuften Texte ist, dass sie an den Leser appellieren, indem sie interessante Themen behandeln und ihr Inhalt sehr aktuell ist. Grammatik- und Satzstruktur sind verständlich. Wir können uns fast alle Strukturen merken. Ich muss sagen, dass ich mit anderen Texten nicht zurechtkommen kann. Ich finde sie recht schwer. Wenn ich zu Hause die Texte selbst lernen möchte, und im Wörterbuch nachschlage, kann ich unbekannte Wörter sogar im Wörterbuch kaum finden. Es fällt mir sehr schwer sowohl Grammatik als auch Wörter zu verstehen. Dennoch sind die Themen nicht interessant. Ich brauche unbedingt eine Erklärung des Lehrers. Aber wenn ich mich zu Hause mit den eingestuften Texten beschäftige, kann ich unbekannte Wörter im Wörterbuch ganz einfach finden und verstehen. Die Erzählform der eingestuften Texte finde ich fließend."

Die Studentin G. A.: „Im Vergleich zu den anderen Texten sind die eingestuften Texte verständlicher. Die Grammatikstruktur ist für unser Niveau angemessen. Aber es gibt viele neue Wörter in jedem Text."

Die Studentin E. Ç: „Die eingestuften Texte sind von anderen Texten, die ich bisher gelesen habe, unterschiedlich. Denn die Grammatik- und Wortstruktur sind verständlich."

In der Forschung ist es auffällig, dass sogar die Student*innen, die sich positiv geäußert haben, die Wörter in den Texten schwer gefunden haben, obwohl sie den Inhalt und die Satzstruktur als verständlich und einfach bezeichnet haben (Auf dieses Dilemma wird im nächsten Kapitel ausführlicher eingegangen).

Der gemeinsame Punkt von drei Student*innen, die den negativen Unterschied zur Sprache gebracht haben, ist der Schwierigkeitsgrad der Wörter. Ein Beispiel davon:

Die Studentin E. N.: „Die eingestuften Texte finde ich schwer und kann sie nicht übersetzen, genauer gesagt; Die Texte auf dem Niveau B1 sind extrem schwer im Vergleich zu den anderen. Die Texte, die ich bisher gelesen habe, sind einfacher und ihre Wörter sind relativ bekannt. Es gibt fast keine unbekannten Wörter." 
Die Studentin F. G.: "Die eingestuften Texte sind im Allgemeinen schwerer als die Texte, die wir in den Seminaren "Lesefertigkeit I und Lesefertigkeit II" bearbeitet haben. Ich kann sie ohne Mühe verstehen. Weil mir die Wörter in den eingestuften Texten schwerfallen, habe ich große Schwierigkeiten beim Verstehen der eingestuften Texte."

Vier Student*innen finden keinen Unterschied zwischen eingestuften Texten und Anderen.

Die Studentin N. T.: „Ich finde, es hat keinen großen Unterschied“

Die Studentin E. K.: „Es gibt keinen Unterschied zwischen den Texten“

Wenn die Student*innenmeinungen analysiert werden, können folgende Schlussfolgerungen gezogen werden;

- $\quad$ Eine Uneinigkeit zwischen den Meinungen macht sich sichtbar. Im Vergleich zu den Texten, die in anderen Vorlesungen bearbeitet worden sind, finden Student*innen, die negative Meinungen äußern, eingestufte Texte schwerer. Die Student*innen, die positive Meinungen äußern, finden eingestufte Texte leichter.

- Obwohl die Experimentalgruppe den getrimmten Mittelwert für 52 auf dem B1 Niveau im Leistungsvortest erreicht hat, ist es erstaunlich, dass sie nach 8-wöchigen Vorlesungen die Texte auf dem B1 Niveau schwer findet.

- Fast die Hälfte der Student*innen in der Experimentalgruppe haben auch ausgesagt, mit vielen unbekannten Wörtern konfrontiert zu sein, obschon die ausgewählten eingestuften Texte für ihr Niveau angemessen sind.

- Weil in anderen Seminaren sowie Lesefertigkeit I, Lesefertigkeit II, erweiterte Lesefertigkeit I und erweiterte Lesefertigkeit II, Textanalyse usw. die ausgewählten Texte nicht in Übereinstimmung mit GeR eingestuft sind, variiert Schwierigkeitsgrad der Texte. Davon ausgehend kann gesagt werden, dass diejenigen, die B1 Texte schwer fanden, in den anderen Seminaren einfache Texte bearbeitet haben müssten. Oder diejenigen, die B1 Texte einfach fanden, müssten in den anderen Seminaren schwierige Texte behandelt haben.

- Die oben erwähnten Textschwierigkeitsgrade in den Seminaren können die Student*innen dazu führen, vor Leseseminaren ernsthafte Hemmungen zu haben. 


\section{Ergebnisse, Diskussionen und Vorschläge}

In diesem Teil werden die Ergebnisse der Forschungsbefunde auf der Basis von eingestuften Texten und diesbezügliche Vorschläge zur Diskussion gestellt.

Aus den Ergebnissen und diesbezüglichen Grafiken ist es zu erkennen, dass im Laufe der Zeit sich keine Überlegenheit zwischen den Gruppen bildete, weil es um eine Fortentwicklung beim Lesen auf Deutsch für jede Gruppe geht. Während es keine Überlegenheit im Vor- und Nachtest gibt, handelt es sich um die Überlegenheit im verzögerten Nachtest zugunsten der Experimentalgruppe. Es kann sich ausdrücken lassen, dass trotz 21 Tage Ferien die Studentin*innen das nicht vergessen haben, was sie in den Vorlesungen gelesen haben. Dieses Ergebnis zeigt, dass die eingestuften Texte im Hinblick des GeR einen grossen Einfluss auf das Behalten des Gelernten im Gedächtnis haben.

Dieses Ergebnis unterstützen ähnliche Forschungen. Sunengin Çakır (2013) hat den Beitrag des GeR in ihrer Dissertation nach den quantitativen und qualitativen Analysen ähnlicherweise bewiesen und die Wichtigkeit des GeR für das Fremdsprachenlernen betont. In ihrer Dissertation bezweckt sie den Einfluss eines Sprachunterrichtsprogramms im Hinblick des GeR auf die Hörverständnisfähigkeit der Studenten auf dem Niveau B1 zu untersuchen. Nach den Forschungsergebnissen ihrer Studie zeigen die Experimentgruppenscoren einen signifikanten Unterschied zugunsten des Nachtests. Mit ihrer Forschung hat Cebeci (2006) vorgeschlagen, bei der Auswahl der Materialien für einen effektiven Fremdsprachenunterricht den Anleitungen und Kriterien des GeR zu folgen. Sie versucht in ihrer Forschung die Effektivität des aufgabenbezogenen Sprachunterrichts auf der Basis von GeR durch Vergleich mit dem traditionellen Sprachunterricht herauszufinden. Nach ihren Forschungsergebnissen war die experimentelle Gruppe im Vokabular deutlich besser als die Kontrollgruppe. İşisağ (2008) hat darauf abgezielt, die Auswirkung des GeR auf die Leistung, Einstellung und die Nachhaltigkeit des Gelernten in den mündlichen Kommunikationsfertigkeiten herauszustellen. Von seinen Ergebnissen ausgehend hat er geäußert, dass die Anwendung von GeR einen neuen und effektiven Ansatz für den Fremdsprachenunterricht bietet, daher kann dieser Ansatz für Fremdsprachenlernende einfach und effektiv sein.

Eines der auffälligsten Ergebnisse der Forschung ist, dass die Experimentalgruppe trotz den 21 tagelangen Ferien, erhebliche Fortschritte für das Lesen auf Deutsch 
verzeichnet hat, obwohl die Kontrollgruppe stagnierte. Auf dieses Ergebnis verweisend kann man feststellen, dass die eingestuften Texte die Student*innen beim Lernen motivieren und anregen, auch in der Freizeit weiter zu lernen. Anders ausgedrückt kann es gesagt werden, dass die eingestuften Texte eine große Rolle bei dem fremdsprachlichen Lernprozess spielen, weil sie ein Motivationsfaktor sind, sowie Yılmaz (2018, S. 1232) in seinem Beitrag betont hat, dass es um verschiedene Faktoren geht, die sich auf Motivation im Fremdsprachenunterricht auswirken und zu diesen Faktoren vor allem Texte zählen, die in den einzelnen Unterrichtsunden behandelt werden.

Die Analysen der Antworten auf die Frage "Gibt es einen Unterschied zwischen den eingestuften Texten und den von Ihnen bisher gelesenen Texten? Wenn ja, was sind die Unterschiede?" wurden in diesem Teil untersucht.

Nach den Analyseergebnissen der schriftlichen Meinungen in der Experimentalgruppe haben die meisten Student*innen (14 von 22 Probanden) positive Einsichten zu den eingestuften Texten entwickelt. Während 4 Probanden ausgesagt haben, dass es keinen Unterschied zwischen den eingestuften und anderen Texten gäbe, fanden andere 4 Probanden die eingestuften Texte hinsichtlich der Grammatik, Vokabeln und Satzstruktur schwer und deswegen unverständlich.

Wie es vorher betont wurde, haben fast alle Student*innen, sogar die positive Einsichten entwickelten Student*innen, zum Ausdruck gebracht, beim Lesen der eingestuften Texte Schwierigkeiten zu haben. In dieser Hinsicht kann es geäußert werden, dass die eingestuften Texte etwas mehr Mühe beanspruchen, wenn auch noch so sie den Niveaus der Student*innen entsprechend ausgewählt wurden.

Von den schriftlichen Meinungen ausgehend kann es gesagt werden, dass die Student*innen eingestufte Texte schwer finden, obwohl sie für ihr Niveau Testergebnissen entsprechend ausgewählt sind. Diese Besonderheit der eingestuften Texte stützt die Theorie „I + 1" von Stephen Krashen (1981) beim Erwerb der zweiten Sprache. Nach dieser Theorie ist es für ein effektives Fremdsprachenlernen erforderlich, dass der Schwierigkeitsgrad der Texte knapp über dem Niveau des Lernenden ist. Im Einklang mit der Theorie von Krashen (1981) kann man ausdrücklich sagen, dass diese Beanspruchung der eingestuften Texte für die akademische Entwicklung der Student*innen notwendig ist und eine große Auswirkung auf den Erwerb der Sprache hat. 


\section{Vorschläge}

In dieser Arbeit wurde nach dem Einfluss der eingestuften Texte im Rahmen des GeR auf die Leistungen und die Nachhaltigkeit von Gelernten der Student*innen geforscht. Basierend auf den Schlussfolgerungen können folgende Punkte vorgeschlagen werden:

1. Wenn man in die Fachliteratur einen Blick wirft, liegen viele Werke im Rahmen des GeR nicht nur in Europa, sondern auch in der Türkei vor. Die meisten dieser Arbeiten fokussieren auf die Kriterien der Bewertung und Beurteilung im GeR oder die Qualifikationen auf der Basis von Kannbeschreibungen der Lehrenden und Lernenden (Siehe nach Deygers \& seinen Kollegen, 2018; Harsch, 2005; Kim, 2006). Deswegen ist es vorzuschlagen, solche Forschungen durchzuführen, die den Beitrag des GeR zu dem Fremdsprachenunterricht messen.

2. Die Lehrpersonen sollten auf den Schwierigkeitsgrad der Texte achten. Die ausgewählten Texte sollten sich dem Niveau der Schüler ausrichten. Jeder Text kann nach den Kriterien des GER adaptiert werden. Denn nach den Ergebnissen der schriftlichen Meinungen können die Texte, die nicht nach den Kriterien vom GeR eingestuft werden, sehr leicht oder sehr schwierig sein.

Die Lehrkraft sollte darauf besonderen Wert legen, eingestufte Texte im Unterricht zu behandeln, weil sie den Sprachlernprozess beschleunigen, und ein Motivationsfaktor für die Lernenden im Fremdsprachenunterricht werden, selbst wenn sie in Bezug auf die Auswahl von Wörtern für schwierig gehalten werden.

3. In der vorgelegten Untersuchung konnten die Texte während der Vorlesungen durch Begleitung der Lehrperson in beschränkten Stunden (50 Minuten für einen Text in einer Vorlesung) behandelt werden. So sind die Textzahlen, die Studenten gelesen haben, auch begrenzt (nur 8 Texte in 8 Wochen). Studien zu den zahlreichen eingestuften Texten durch freizeitliches Lesen können durchgeführt werden, um den Einfluss der eingestuften Texte klarer vor Auge zu stellen.

4. In dieser Studie wurde nur der Einfluss des GeR auf die Lesefähigkeit untersucht. Weitere Studien einschließlich anderer Sprachfertigkeiten sollen durchgeführt werden, um die Sprache als Ganzes zu erfassen. Auf diese Weise kann sich der Einfluss des GeR auf den Sprachunterricht herauskristallisieren. 
Begutachtung: Extern begutachtet.

Interessenkonflikt: Es besteht kein Interessenkonflikt.

Finanzielle Förderung: Dieser Beitrag basiert auf Dissertation, die von der Abteilung für die wissenschaftlichen Forschungsprojekte der Universität Necmettin Erbakan mit dem Projekt, nummeriert 161410002, finanziell unterstützt wurde.

Peer-review: Externally peer-reviewed.

Conflict of Interest: The authors have no conflict of interest to declare.

Grant Support: This Article is based on dissertation which was supported by the Department of Scientific Research projects of the University of Necmettin Erbakan (Project Number: 161410002).

\section{Literaturverzeichnis}

Burkhart, M. \& Sedlmeier, P. (2015). Explorative und deskriptive Datenanalyse mit R (Sozialwissenschaftliche Forschungsmethoden) Taschenbuch. Münschen und Mering: Rainer Hamp Verlag.

Büyüköztürk, Ş., Kılıç Ç. E., Akgün, Ö. E., Karadeniz, Ş. \& Demirel, F. (2012). Bilimsel Araştırma Yöntemleri (13. baskı). Ankara: Pegem Akademi Yayınları.

Cebeci, N. (2006). The Effectivity Of Task-Based Activities On Vocabulary Competence Designed In Accordance With The Common European Framework. Master Thesis, Trakya University Institute of Social Sciences, Edirne.

Deygers, B., Zeidler, B., Vilcu, D., Carlsen, C. H. (2018). One Framework to Unite Them All? Use of the CEFR in European University Entrance Policies. Language Assessment Quarterly, 15, 3-15.

Dzaldov, B. S. \& Petersen S. (2005) Book Leveling and Readers. The Reading Teacher 59(3), 222-229. DOI: 10.1598/ RT.59.3.2

Gemeinsamer Europäischer Referenzrahmen für Sprachen: Lernen, Lehren, Beurteilen. http://student.unifr.ch/ pluriling/assets/files/Referenzrahmen2001.pdf Zugriffsdatum: 16.04.2017.

George, D. \& Mallery, P. (2010). SPSS für Windows Schritt für Schritt: Eine einfache Anleitung und Referenz, 17.0 Update (10a ed.) Boston: Pearson.

Glaswell, K. \& Ford, P. M. (2010). Teaching Flexibly With Leveled Texts: More Power for Your Reading Block. The Reading Teacher, 64(1), 57-60. DOI: 10.1598/RT.64.1.7

Harsch, C. (2005). Der Gemeinsame Europäische Referenzrahmen für Sprachen: Leistung und Grenzen. Die Bedeutung des Referenzrahmens im Kontext der Beurteilung von Sprachvermögen am Beispiel des Semikreativen Schreibens im DESI- Projekt, https://opus.bibliothek.uniaugsburg.de/opus4/frontdoor/ deliver/index/docld/297/file/DISS_Claudia_Harsch.pdf.Zugriffsdatum:16.03.2018.

İşisağ, K. U. (2008). Sözlü Illetişim Becerilerinin Gelişiminde Diller İçin Avrupa Ortak Başvuru Metni Uygulamalarının Başarıya, Tutuma Ve Kalıcılığa Etkisi. Doktora tezi, Ankara Üniversitesi Sosyal Bilimler Enstitüsü, Ankara.

Karasar, N. (1994). Araştırmalarda Rapor Hazırlama (7. Basım). Ankara: Araştırma Eğitim Danışmanlık LTD.

Kim, H. (2006). Gemeinsamer europäischer Referenzrahmen für Sprachen: lernen, lehren, beurteilen als Orientierung für den Fremdsprachenunterricht in Korea, Online: http://www.goethe.de/z/50/commeuro/Zugriffsdatum: 20.03.2017. 
Krashen, S. D. (1981). Second Language Acquisition and Second Language Learning. University of Southern California: Pergamon Press Inc.

Kuwan, H. \& Waschbüsch, E. (1999). Delphi Befragung 1996/1998 -Abschlussbericht zum "Bildungs-Delphi", München.

Meyer, H. (1987). Handlungsorientierter, handelnder und schülerorientierter Unterricht. (Hilbert Meyer) Unterrichtsmethoden (Band 1). Theorieband. Frankfurt am Main: Cornelsen Scriptor.

Sönmez, V. \& Alacapınar, F. G. (2014). Örneklendirilmiş Bilimsel Araştırma Yöntemleri (3. Baskı). Ankara: Anı Yayıncılık.

Sunengin, Ş. Z. (2013). Fransızca Öğretiminde Avrupa Ortak Başvuru Metni Uygulamalarının Dinlediğini Anlama Becerisine Etkisi. Doktora Tezi, Ankara Üniversitesi Sosyal Bilimler Enstitüsü, Ankara.

Yıldırım, A. \& Şimşek H. (2008). Sosyal Bilimlerde Nitel Arastirma Yöntemleri. Ankara: Seckin.

Yılmaz, H. (2018). Die Rolle Der Texte Im Fremdsprachlichen Deutschunterricht. Abant İzet Baysal Üniversitesi Eğitim Fakültesi Dergisi, 18 (2), 1231-1243. DOI: 10.17240/aibuefd.2018..-431519. 